Research Paper

\title{
Analysis Of The KLIC-score; An Outcome Predictor Tool For Prosthetic Joint Infections Treated With Debridement, Antibiotics And Implant Retention
}

\author{
Sean DX Duffy ${ }^{\bowtie}$, Nathanael Ahearn, Elizabeth SR Darley, Andrew J Porteous, James R Murray, Nicholas R \\ Howells \\ Avon Orthopaedic Centre, Southmead Hospital, Bristol \\ $\triangle$ Corresponding author: Address: Trauma \& Orthopaedic Department, Level 6 Office, Southmead Hospital, Dorian Way, Westbury-on-Trym, Bristol, BS10 \\ 5NB. E: seandxduffy@gmail.com; T: 07764575948 \\ (c) Ivyspring International Publisher. This is an open access article distributed under the terms of the Creative Commons Attribution (CC BY-NC) license \\ (https://creativecommons.org/licenses/by-nc/4.0/). See http://ivyspring.com/terms for full terms and conditions.
}

Received: 2017.07.10; Accepted: 2017.12.09; Published: 2018.07.27

\begin{abstract}
Background: Debridement, antibiotics and implant retention (DAIR) forms the primary treatment modality for early prosthetic joint infection (PJI). The KLIC score has been proposed as a risk stratification tool for use in predicting outcome of prosthetic knee infections. Our aim was to determine the accuracy of this scoring system at an independent tertiary PJI centre in a typical DAIR population.

Methods: Between 2008 and 2015, patients with infected knee prostheses treated with DAIR were identified. The patient notes and blood tests were reviewed retrospectively and the 'KLIC-score' was calculated and correlated with outcome. The end point for early failure was defined as: 1) the need for unscheduled surgery, 2) infection-related death $\leq 12$ months from debridement or 3) the need for suppressive antibiotic treatment.

Results: 59 patients received DAIR procedures for knee PJI. Treatment was successful in 41 patients (69\%) with early failure in 18 patients (31\%). Patients deemed high-risk (KLIC-score $\geq 7$ ) had notably higher failure rates $(60 \%)$ than those scoring $<7(28 \%)$. No relationship can be drawn between KLIC-scores of $<7$ and failure rates.

Conclusions: The KLIC-score applied retrospectively was able to predict patients with the highest risk of early failure but provides little information in patients with scores of $<7$.
\end{abstract}

Key words: Prosthetic, Joint, Infection, Outcome, KLIC, Tool, DAIR

\section{Introduction}

In 2015/16, 104,695 knee arthroplasty operations were performed in the UK (1). Whilst only a minority of joints become infected, prosthetic joint infection (PJI) is a potentially devastating complication, occurring in $0.6 \%$ of patients undergoing total knee arthroplasty (TKA) (2). Infection is the second most common cause for arthroplasty revision (2). TKA numbers are increasing annually, with a predicted rise of $673 \%$ in TKA demand in the United States by 2030 (3), and the burden of PJI will rise accordingly. The treatment of PJI requires infection eradication to enable the patient to retain walking ability and optimal knee function.
Treatment options for PJI include antibiotic suppression, arthroplasty resection, one or two-stage revision arthroplasty, arthrodesis, amputation, and attempts at implant retention. The ability to treat PJI with debridement, antibiotics, and implant retention (DAIR) is an attractive proposition for a number of reasons. It is a single operation, which negates the need for the patient to undergo a staged procedure, if successful is a less expensive treatment than a two-staged procedure, and is associated with a shorter period of disability. DAIR is indicated in early post-operative infections, considered to be within 3 months of index procedure and symptom duration of less than 3 weeks. Some centres have extended the 
indications for DAIR for any well fixed, well-aligned knee with acute infection, irrespective of timing from index surgery (4).

Contraindications to DAIR include the presence of a sinus tract, inability to close the wound and a loose prosthesis (5). Two-staged arthroplasty revision remains the gold-standard treatment with success rates of over $80 \%$ (6-8). PJI treatment with DAIR has shown variable success rates, ranging from $32-100 \%$, with improved results associated with select patient groups (4, 9-14). The ability to accurately predict poor outcome and identify such patients would be a useful tool in avoiding unsuccessful procedures in an attempt to combat PJI. In addition this would avoid the financial burden of procedure which is likely to fail and require 1 or 2 stage revision TKA.

A scoring system developed by Tornero et al. identified various factors that could be used to predict the outcome of DAIR in PJI following hip or knee arthroplasties (15). The KLIC-score (ranging from 0 to 9.5), takes into account patient kidney function, liver function, index surgery, cemented prosthesis and c-reactive protein (CRP) value (Table 1). It has not yet been validated in other patient populations or in other units that perform DAIR procedures to treat PJI. The aim of this study was to determine the accuracy of this scoring system for use in predicting outcome in prosthetic knee infections for our patient population.

Table 1: KLIC-score, as described by Tornero et al.

\begin{tabular}{lll}
\hline Variable & & Score \\
\hline $\mathrm{K}$ & Chronic Renal Failure (Kidney) & 2 \\
$\mathrm{~L}$ & Liver Failure & 1.5 \\
$\mathrm{I}$ & Index surgery $=$ & 1.5 \\
& Revision surgery & \\
& or prosthesis to treat femoral neck fracture & \\
$\mathrm{C}$ & Cemented prosthesis & 2 \\
$\mathrm{C}$ & C-reactive protein $(\mathrm{CRP}>115 \mathrm{mg} / \mathrm{L})$ & 2.5 \\
& Total & 9.5 \\
\hline
\end{tabular}

\section{Patients and Methods}

This retrospective cohort study was undertaken at a combined microbiology and orthopaedic regional tertiary referral centre for PJI. All PJI patients are managed through a combined multidisciplinary PJI clinic service. Patients who had a DAIR procedure between June 2008 and September 2015 were identified from a prospectively collected PJI database.

Inclusion criterion was any patient with an infected knee prosthesis treated with DAIR at our institution in the given time period. Infection was defined as per international consensus definition (2014) as two positive periprosthetic cultures with phenotypically identical organisms, or a sinus tract communicating with the joint, or three of the following criteria: elevated CRP and ESR, elevated WBC or positive change on leukocyte esterase strip, elevated synovial fluid polymorph count (not available in our unit as an absolute value), a single positive culture or positive histological analysis of periprosthetic tissue (5). This definition was the most up to date and was therefore chosen. It differs from that used by Tornero et al. in that to diagnose PJI, three rather than four of the criteria described are required. This had no effect on the number of patients included. We considered pre-operative cultures only, as this is the information available at the time the KLIC-score is intended to be used. There were no exclusions as this represents a consecutive series of patients. The rationale for these criteria was to assess the KLIC score in a typical DAIR population and not confine it only to early acute infections. Online patient records, including microbiology results and pathology blood test results, were used to retrospectively assign a KLIC-score, using the most recent pre-operative data, to each patient that received a DAIR procedure and correlated with outcome. All patients had sufficient information available to calculate the KLIC-score. The primary outcome measure was early success of the DAIR procedure, defined as no end points for early failure within the first 12 months. The end point for early failure was defined as: 1) the need for unscheduled surgery within the first 12 months, 2) death related to infection within the first 12 months or 3) the need for long-term suppressive antibiotic treatment in patients unsuitable for further surgery.

\section{Organism identification and standard treatment}

Pre-operative aspiration was undertaken immediately on presentation and samples of fluid or tissue submitted for microscopy and culture according to standard procedure. Enrichment cultures were incubated for 5 days, susceptibilities were performed according to the British Society for Antimicrobial Chemotherapy (BSAC) methodology (16). 48-72 hours were waited to obtain results from early initial culture. The DAIR was not delayed until time of enrichment culture in view of the acute need to proceed with surgery. If however an early microbiology result was available targeted intraarticular antibiotics would be used mixed into calcium sulphate delivery pellets.

Following DAIR, standard treatment was to initiate intravenous vancomycin and oral rifampicin pending culture results unless Gram negative organism had been cultured at aspiration, in which case empirical antibiotics were modified accordingly. Antibiotics treatment was rationalized when enrichment culture results became available. A standard approach would be approximately 5-10 days 
of intravenous therapy followed by approximately 5-6 months or oral antibiotics, but varied according to patient factors and progress.

Online pathology records and outpatient clinic letters were used to determine whether the aetiological organism was known prior to the DAIR procedure.

\section{Statistical analysis}

Logistic regression analysis was performed with duration of symptoms, time from index procedure, microorganism identified and the components of the KLIC-score as co-factors. The differences between the individual KLIC-score groups were performed using a Mann-Whitney test and Kruskal-Wallis test. A p-value $<0.05$ was considered to be statistically significant. All statistical analyses were performed using SPSS 23 (IBM Corp, Armonk, New York).

\section{Results}

A total of 59 patients were identified who met the inclusion criterion, receiving DAIR procedures for prosthetic knee infections. The median age was 71 (IQR 17) for the male patients $(n=34)$ and 73 (IQR 10) for the female patients $(n=25)$. The median duration of follow up was 2.25 years (IQR 1.58) with a minimum follow up of 12 months. The original joint arthroplasty type was TKA in 55 patients (93\%), unicompartmental knee arthroplasty (UKA) in 2 patients (3\%) and patellofemoral joint replacement (PFJR) in 2 patients (3\%). The median time from index procedure to DAIR was 7 months (IQR 27), ranging from 11 days to 10.5 years. The median duration of symptoms prior to DAIR procedure was 6 days (IQR 10). In four cases, the duration of symptoms prior to DAIR was $>60$ days, and in one case $>200$ days. In the latter, a DAIR was performed following a period of acute worsening of chronic infective symptoms, which had previously almost completely subsided. However in these five cases, which did not meet accepted duration for DAIR procedure, four failed early. Additionally as would be expected, in early infections (symptom duration $<3$ weeks) failure rate was less than that seen in late-acute infections $(>3$ months from index procedure), $19 \%$ (mean KLIC 4.5 ) and $28 \%$ (mean KLIC 5.5) respectively.

In total there were 41 patients $(69 \%)$ in whom the DAIR procedure was defined as successful, with early failure in 18 patients (31\%) (Table 2). Interestingly in this series all patients that failed DAIR had done so within 60 days from procedure with no further failures out to a median follow up of 1.25 years. Patients in the 'high risk' KLIC group ( $\geq 7$ points) had a failure rate of $60 \%$. Patients in the 'low risk' KLIC group ( $\leq 2$ points) had a failure rate of $33 \%$.
Table 2: KLIC-score result and treatment outcomes

\begin{tabular}{lllll}
\hline KLIC-score & Group size & Failure No. & Failure rate (\%) & Success rate (\%) \\
\hline$\leq 2$ & 12 & 4 & $33 \%$ & $67 \%$ \\
$2.5-3.5$ & 7 & 2 & $29 \%$ & $72 \%$ \\
$4-5$ & 19 & 4 & $21 \%$ & $79 \%$ \\
$5.5-6.5$ & 16 & 5 & $31 \%$ & $69 \%$ \\
$\geq 7$ & 5 & 3 & $60 \%$ & $40 \%$ \\
TOTALS & $\mathbf{5 9}$ & $\mathbf{1 8}$ & $\mathbf{3 1} \%$ & $\mathbf{6 9} \%$ \\
\hline
\end{tabular}

In 53 cases $(90 \%)$, the aetiological microorganism had been identified prior to debridement. The remaining $6(10 \%)$ cases underwent DAIR procedure without an identifiable organism. The vast majority of patients $(85 \%)$ grew the same microorganism from samples prior to and during the DAIR procedure. In 4 cases, gram-negative organisms were isolated, much fewer than in the original paper. Gram-positive organisms were isolated in 43 cases. Staphylococcus species was isolated in 33 joints and streptococcus species in 13 joints (6.8\%). Four cases were treated as polymicrobial, ie $>1$ organism cultured and considered significant (17). Among the patients where an organism was identified, the most commonly isolated organism was Staphylococcus aureus (SA) $(n=21)$, one case was polymicrobial. The second most frequently isolated organism was coagulase-negative staphylococcus (CoNS) ( $\mathrm{n}=12)$, one case was polymicrobial. Staphylococcal PJI had an overall failure rate of $25 \%(n=8)$. Individual staphylococcal species had varied results, the highest failure rate was seen in $S A$ infections of $35 \%(n=7)$. CoNS infections had a much lower failure rate of $9 \%(n=1)$. Streptococcal infections had an overall failure rate of $23 \%(n=3)$, with each of these failures observed in patients with Group G beta-haemolytic Streptococcal infection. Other microorganisms were isolated in 7 patients, including Enterobacter cloacae $(\mathrm{n}=1)$, Enterococcus faecalis $(\mathrm{n}=1)$, Escherichia coli $(\mathrm{n}=2)$, Citrobacter species $(\mathrm{n}=2)$ and Pseudomonas aeruginosa $(\mathrm{n}=1)$.

Interestingly, exclusion of the patients in whom cultures were negative or not attempted prior to performing a DAIR procedure appears to enhance the scores reliability and sensitivity in identifying the highest and lowest risk patient groups. We will consider further evaluation of this modification.

\section{Statistical analysis}

There was no significant statistical difference between the various KLIC-score groups ( $p=0.77$, Chi Squared). Comparing the outcome of the patients with a KLIC-score $\geq 7$ with those $<7$ again showed no significant statistical difference ( $p=0.24$, Chi Squared). No other factors including duration of symptoms prior to DAIR were found to have a significant difference on outcome. $p=0.14-0.61$ ). If only culture positive cases were included in analysis then the KLIC score became more sensitive with failures in $75 \%$ of 
cases with a score $\geq 7$ and a statistically significant difference between outcomes for patients with a KLIC score $\geq 7$ and $<7$ ( $p=0.032$, Chi Squared Test).

\section{Discussion}

PJI following knee arthroplasty poses a significant problem to both patients and surgeons. The ability to predict outcome of DAIR procedures would be a valuable tool in targeting treatment in susceptible patients. This study has demonstrated the KLIC-score, as devised by Tornero et al. is able to detect the group of patients with the highest rate of early failure (score $\geq 7$ points) following DAIR, in patients with infected knee prosthesis, when applied retrospectively. However, there was no correlation between low scores and treatment outcome. As such, the KLIC-score as a tool for predicting DAIR outcome is useful in predicting early failure, but only in patients that score very highly. In our cohort, 18 (31\%) cases experienced early failure post DAIR; including one case undergoing DAIR $>200$ days post diagnosis of infection, an overall success rate of $69 \%$. This success rate is comparable with the rates described by other units, ranging from $32-100 \%(4,9-14)$. One-stage revision success rates have been reported as high as $86 \%$ (9). However, in a study conducted on over 16,600 TKA PJI, one-stage revisions had a reported risk of re-infection one-third greater than two-stage procedures (6). Two-stage procedures have proved to be the most successful at infection eradication $(18,19)$, but have the disadvantages of increased expense, multiple operations and a prolonged duration of reduced mobility. In addition, when used as a final attempt at infection eradication post a failed DAIR procedure, this has proved less effective in some studies (20). The less invasive and less expensive option of performing DAIR is an attractive proposition, when considered likely to be successful.

Risk factors for treatment failure are well described in the current literature and fall into host-related, bacterial-related and treatment-related groups (Table 3). Such factors include: sinus tract at time of DAIR $(12,21)$, long duration of symptoms prior to debridement $(12,22,23)$ and infection with Staphylococcus aureus $(4,12,23,24,25)$. Kuiper et al. reported high failure rates associated with rheumatoid arthritis, late infection ( $>2$ years after arthroplasty), ESR above $60 \mathrm{~mm} / \mathrm{h}$ at presentation and CoNS infection (10). In contrast, in this study CoNS-associated PJI had a high success rate of $91 \%$, comparable to the rates described by Peel et al. (26), but this may reflect the strain variability and susceptibility profile of organisms at different institutions. Importantly, there is variation in treatments and definitions of success and failure between the studies (Table 3) correlation between the risk factors described should take this into account.

Factors most commonly associated with treatment failure differ from one unit to another. Identification of the presence of such factors by surgeons is important in minimising treatment failure. This may be as a result of opting for alternative treatments/procedures in high risk patients or by targeted patient optimisation pre-operatively. A predictor tool must take this into account so that it may prove reliable across different patient populations.

In general, most studies describe a collection of statistically significant factors associated with negative or positive outcome post DAIR. Besides the KLIC-score, there is only one other similar predictive tool in the current literature. Buller et al. describe a nomogram for prediction of treatment success pre-operatively which contains 17 factors (27). This nomogram does not exclude patients with negative cultures pre-operatively, a situation that is not uncommonly seen. Both the KLIC-score and the nomogram described by Buller et al. are designed on data from hip and knee arthroplasty infections.

This study suggests that the KLIC-score is not useful at distinguishing between higher and lower risk patients when scoring $<7$ points. It is not possible to further stratify score below 7, although all such groups are at an overall lower risk of failure (21-33\%). Scoring $\geq 7$ points on the KLIC-score equates to a high risk of failure $(60 \%)$. In the latter patient group, the tool may therefore be useful in identifying patients at higher risk of failure. In such patients, surgeons may instead opt for a two-staged revision based on this information or be able to discuss the higher risk of failure with the patient.

This study has some limitations. As above, our study was retrospective and some data was not available. As a tertiary referral centre, a proportion of our patients may have received variable initial investigations and management at other centres which affect diagnosis and outcome. This however is the nature of PJI management and increasingly care is moving towards a model whereby PJI care is coordinated from a regional centre. The study is also limited by the small number of patients scoring $\geq 7$. This is partly due to the parameters of the scoring system, and the original use in both hip and knee arthroplasty. In two cases the exact date of the index procedure was unknown but the month and year or sometimes only year was recorded. In five cases the date of symptom onset was unknown. Finally, in six cases the infecting organism was unknown. Comparatively, in the original study all patients were reported to have positive cultures. 
Table 3: Summary of risk factors associated with failure after DAIR in the current literature

\begin{tabular}{|c|c|c|c|c|c|c|c|c|c|c|c|c|c|}
\hline Risk Factor & 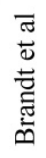 & 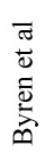 & 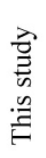 & 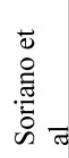 & 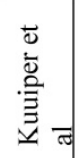 & 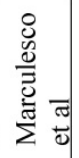 & $\begin{array}{l}\bar{\pi} \\
\frac{\pi}{0} \\
\frac{0}{8} \\
2\end{array}$ & 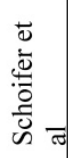 & 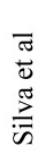 & 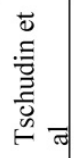 & 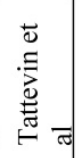 & 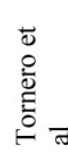 & 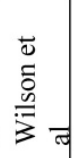 \\
\hline $\begin{array}{l}\text { Staphylococcus } \\
\text { aureus }\end{array}$ & $\checkmark$ & $\checkmark$ & $\checkmark$ & $\checkmark$ & & $\checkmark$ & & $\checkmark$ & & & & & $\checkmark$ \\
\hline $\begin{array}{l}\text { Long duration } \\
\text { of symptoms }\end{array}$ & $\checkmark$ & & & & & $\checkmark$ & & & & & $\checkmark$ & & \\
\hline $\begin{array}{l}\text { Revision } \\
\text { arthroplasty }\end{array}$ & $\checkmark$ & $\checkmark$ & & & & & & & & & & & \\
\hline $\begin{array}{l}\text { Hinged } \\
\text { arthroplasty }\end{array}$ & & & & & & & & $\checkmark$ & $\checkmark$ & & & & \\
\hline Sinus Tract & & & & & & $\checkmark$ & & & $\checkmark$ & & & & \\
\hline $\begin{array}{l}\text { Rheumatoid } \\
\text { arthritis }\end{array}$ & & & & & $\checkmark$ & & & & $\checkmark$ & & & & \\
\hline Late infection & & & & & $\checkmark$ & & & & & & $\checkmark$ & & \\
\hline $\begin{array}{l}\text { KLIC } \geq 7 \\
\text { points }\end{array}$ & & & $\checkmark$ & & & & & & & & & $\checkmark$ & \\
\hline CoNS & & & & & $\checkmark$ & & & & & & & & \\
\hline$M R S A$ & & & & & & & $\checkmark$ & & & & & & \\
\hline $\begin{array}{l}<90 \text { days } \\
\text { antibiotics }\end{array}$ & & & & & & & $\checkmark$ & & & & & & \\
\hline $\begin{array}{l}\text { Group G } \\
\text { Streptococcus }\end{array}$ & & & $\checkmark$ & & & & & & & & & & \\
\hline Loose implant & & & & & & & & & & $\checkmark$ & & & \\
\hline Advanced age & & & & & & & & & & & $\checkmark$ & & \\
\hline $\begin{array}{l}\text { ESR } \\
>60 \mathrm{~mm} / \mathrm{hr}\end{array}$ & & & & & $\checkmark$ & & & & & & & & \\
\hline $\begin{array}{l}\text { Arthroscopic } \\
\text { debridement }\end{array}$ & & $\checkmark$ & & & & & & & & & & & \\
\hline $\begin{array}{l}\text { Single Surgical } \\
\text { Debridement }\end{array}$ & & & & & & & $\checkmark$ & & & & & & \\
\hline $\begin{array}{l}\geq 4 \text { surgical } \\
\text { debridements }\end{array}$ & & & & & & & $\checkmark$ & & & & & & \\
\hline
\end{tabular}

\section{Conclusions}

A reliable outcome predictor tool would be of huge benefit in terms of morbidity and cost reduction when treating PJI. High failure rates have been associated with DAIR in the literature and there is concern that a failed DAIR has a negative impact on the success of salvage two-staged procedures thereafter. As such, identifying patients with high chance of a successful treatment outcome and importantly those much less likely to have a successful outcome, would provide a significant advantage to patient, surgeon and health economy. Use of the KLIC-score in our cohort of patients has shown it to be useful in predicting poor outcome in those that scored $\geq 7$ points, the group with the highest failure rate.

Further investigation of the modification of the score may improve it's reliability and use in this patient cohort.

\section{Supplementary Material}

Supplementary Appendix 1.1. http://www.jbji.net/v03p0150s1.pdf

\section{Competing Interests}

The authors have declared that no competing interest exists.

\section{References}

1. [Internet] National Joint Registry, 13th Annual Report 2016. NJR Reports. 2016. (Available online at www.njrcentre.org.uk)

2. Public Health England. Surgical site infections (SSI) surveillance: NHS hospitals in England. 2014/15. London:Public Health England, December 2015.

3. Kurtz SM, Lau E et al. Infection Burden for Hip and Knee Arthroplasty in the United States. J Arthroplasty 2008;23:984-91.

4. Byren I, Bejon P et al. One Hundred and Twelve Infected Arthroplasties Treated With 'Dair' (Debridement Antibiotics and Implant Retention): Antibiotic Duration and Outcome. J Antimicrob Chemother 2009;63:1264-71

5. Parvizi J and Gehrke T. International Consensus on Periprosthetic Joint Infection: Let Cumulative Wisdom be a Guide. J Bone Joint Surg Am 2014;96:441

6. Cochran AR, Ong KL et al. Risk of Reinfection After Treatment of Infected Total Knee Arthroplasty. J Arthroplasty 2016;31:156-61 
7. Haleem AA, Berry DJ and Hansen AD. Mid-Term to Long-Term Followup of Two-Stage Reimplantation for Infected Total Knee Arthroplasty. Clin Orthop Relat Res 2004;428:35-39

8. Goldman RT, Scuderi GR and Insall JN. 2-Stage Reimplantation for Infected Total Knee Replacement. Clin Orthop Relat Res 1996;331:118-24

9. Silva M, Tharani R and Schmalzried TP. Results of Direct Exchange or Debridement of the Infected Total Knee Arthroplasty. Clin Orthop Relat Res 2002;404:125-31

10. Kuiper JW, Vos SJ et al. Prosthetic Joint-Associated Infections Treated With Dair (Debridement Antibiotics Irrigation and Retention): Analysis of Risk Factors and Local Antibiotic Carriers in 91 Patients. Acta Orthop 2013;84:380-6

11. Lora-Tamayo J, Murillo $\mathrm{O}$ et al. A Large Multicenter Study of Methicillin-Susceptible and Methicillin-Resistant Staphylococcus Aureus Prosthetic Joint Infections Managed With Implant Retention. Clin Infect Dis 2013;56:182-194

12. Marculescu CE, Berbari EF et al. Outcome of Prosthetic Joint Infections Treated With Debridement and Retention of Components. Clin Infect Dis 2006;42:471-78

13. Meehan AM, Osmon DR et al. Outcome of Penicillin-Susceptible Streptococcal Prosthetic Joint Infection Treated With Debridement and Retention of the Prosthesis. Clin Infect Dis 2003;36:845-49

14. Zimmerli W, Widmer AF et al. Role of Rifampin for Treatment of Orthopedic Implant-Related Staphylococcal Infections: A Randomized Controlled Trial. Foreign-Body Infection (Fbi) Study Group. JAMA 1998;279:1537-41

15. Tornero E, Morata L et al. Klic-Score for Predicting Early Failure in Prosthetic Joint Infections Treated With Debridement Implant Retention and Antibiotics. Clin Microbiol Infect 2015;21:786.e9-786.e17

16. [No authors listed] BSAC methodology for Antimicrobial Sensitivity testing. The British Society for Antimicrobial Chemotherapy. 2015;14

17. Atkins BL, Athanasou $\mathrm{N}$ et al. Prospective Evaluation of Criteria for Microbiological Diagnosis of Prosthetic-Joint Infection At Revision Arthroplasty. The Osiris Collaborative Study Group. J Clin Microbiol 1998;36:2932-9

18. Puhto AP, Puhto TM et al. Two-Stage Revision for Prosthetic Joint Infection: Outcome and Role of Reimplantation Microbiology in 107 Cases. J Arthroplasty 2014;29:1101-4

19. Evans RP. Successful Treatment of Total Hip and Knee Infection With Articulating Antibiotic Components: A Modified Treatment Method. Clin Orthop Relat Res 2004;427:37-46

20. Sherrell JC, Fehring TK et al. The Chitranjan Ranawat Award: Fate of Two-Stage Reimplantation After Failed Irrigation and Débridement for Periprosthetic Knee Infection. Clin Orthop Relat Res 2011;469:18-25

21. Tschudin-Sutter S, Frei R et al. Validation of a Treatment Algorithm for Orthopaedic Implant-Related Infections With Device-Retention-results From a Prospective Observational Cohort Study. Clin Microbiol Infect 2016:22:457.e1-9

22. Tattevin P, Crémieux AC et al. Prosthetic Joint Infection: When Can Prosthesis Salvage be Considered. Clin Infect Dis 1999;29:292-95

23. Brandt CM, Sistrunk WW et al. Staphylococcus Aureus Prosthetic Joint Infection Treated With Debridement and Prosthesis Retention. Clin Infect Dis 1997;24:914-19

24. Schoifet SD and Morrey BF. Treatment of Infection After Total Knee Arthroplasty By Débridement With Retention of the Components. J Bone Joint Surg Am 1990;72:1383-90

25. Wilson MG, Kelley K and Thornhill TS. Infection as a Complication of Total Knee-Replacement Arthroplasty. Risk Factors and Treatment in Sixty-Seven Cases. J Bone Joint Surg Am 1990;72:878-83

26. Peel TN, Buising KL et al. Outcome of Debridement and Retention in Prosthetic Joint Infections By Methicillin-Resistant Staphylococci With Special Reference to Rifampin and Fusidic Acid Combination Therapy. Antimicrob Agents Chemother 2013;57:350-5

27. Buller LT, Sabry FY et al. The Preoperative Prediction of Success Following Irrigation and Debridement With Polyethylene Exchange for Hip and Knee Prosthetic Joint Infections. J Arthroplasty 2012;27:857-64.e1-4 